Running head: PURPOSES OF ONLINE MEMORY SHARING SCALE

\title{
Creation of the Purposes of Online Memory Sharing Scale
}

\author{
Qi Wang
}

Cornell University

Correspondence should be addressed to Qi Wang, Department of Human Development, Cornell University, MVR Hall, Ithaca, NY 14853-4401; telephone 607-255-9376; electronic mail qiwang@cornell.edu. 


\begin{abstract}
I describe in this article the creation of the Purposes of Online Memory Sharing Scale (POMSS). I provide an overview of the theoretical and empirical backgrounds against which the scale was developed. I then present data from a pilot sample which indicate that the scale as a whole is a reliable measure of the reasons for people to share their experiences online. In addition, three of the subscales - Self, Social, and Therapeutic - had acceptable to excellent internal consistency reliability, while the Directive subscale had poor internal consistency and should be used with caution. The various purposes of online memory sharing were positively associated with the frequency of the sharing activity, which lends support for the validity of the scale. The Purposes of Online Memory Sharing Scale (POMSS) provides a useful tool for researchers to examine memory expression and communication in the digital age.
\end{abstract}

Keywords: Memory sharing; Social media; Internet; Memory functions; Measurement; Scale; Reliability; Validity 


\section{Creation of the Purposes of Online Memory Sharing Scale}

Memory sharing is a common everyday activity. We share memories with others to convey information about who we are. We share memories to entertain others and to develop and maintain relationship closeness. We also share memories of negative experiences to gain empathy and support and to solicit advice from others. We further share memories to impart lessons we have learned from the past and to help others. These purposes of memory sharing for self, social, therapeutic, and directive functions have been documented in extensive empirical research (e.g., Alea \& Bluck, 2003; Bluck, 2003; Kulkofsky, Wang, \& Hou, 2010; Pillemer, 1998; Wang, Koh, Song, \& Hou, 2015). Importantly, in the contemporary digitally mediated society, sharing memory is no longer limited to face-to-face interactions but frequently takes place online, via social media. Does online memory sharing serve similar purposes as memory sharing in person?

Based on data from a large survey by the Pew Internet and American Life Project with a nationally representative sample of bloggers (Lenhart \& Fox, 2006), Wang (2013) suggests that the major reasons provided by the participants for their sharing personal experiences online can be sorted into three broad functions of self, social, and directive. This observation is confirmed by findings from communication research on motives for personal blogging (Hollenbaugh, 2011; Lee, Im, \& Taylor, 2008; Schmitt, Dayanim, \& Matthias, 2008). In addition to the three functions, the extant research further suggests that sharing memories online is also used for the therapeutic purpose of emotion regulation and coping (Baker \& Moore, 2011; Nardi, Schiano, \& Gumbrecht, 2004). Thus, online memory sharing appears to serve similar purposes as memory sharing in person. Several scales have been developed to measure the reasons for online personal 
blogging or macroblogging (e.g., Baker \& Moore, 2011; Hollenbaugh, 2011). However, these scales may not be pertinent to online memory sharing more generally, especially memory sharing via microblogging such as status updates, which has become a more prominent form of online communication particularly among teens and young adults (Lenhart, Purcell, Smith, \& Zichuhr, 2010).

To better understand the reasons for which people share their experiences online regardless of the social media outlet, I developed a 20-item Purposes of Online Memory Sharing Scale (POMSS). The scale was constructed in reference to prior research on motives for personal blogging (Baker \& Moore, 2011; Hollenbaugh, 2011; Lee et al., 2008; Lenhart \& Fox, 2006) as well as the memory function literature (Alea \& Bluck, 2003; Bluck, 2003; Kulkofsky et al., 2010; Pillemer, 1998; Wang et al., 2015). It consists of four subscales - Self, Social, Therapeutic, and Directive, which include 5 items, 6 items, 5 items, and 4 items, respectively. The Social purpose subscale contains more items than others given the prominent social functions of sharing memories with others (Alea \& Bluck, 2003; Guan \& Wang, 2020; Kulkofsky et al., 2010). The scale is provided in Appendix A. The subscales and their items are listed in Table 1.

The scale was pilot-tested with 31 college students $($ Mean Age $=20.76$ years, $S D=.92$ ), including 24 females and 7 males, as part of a larger study that investigated the effect of online memory sharing on memory retention (Wang, Lee, \& Hou, 2017). Participants were asked to indicate how much each item described their reasons for sharing experiences online by choosing a response from 1 ("'not at all”) to 5 ("exactly my reasons"). Participants also kept a daily diary for a week in which they recorded all the events that happened to them on each day and reported whether they shared any of the events online. The total number of memories participants shared online over the week was tallied. 
Table 1. Purposes of Online Memory Sharing Scale (POMSS)

Subscale

Self

Cronbach's $\alpha=.735$
Item

To express myself

To document my personal experiences

To get attention

To record my thoughts and feelings so I can reflect on them

To communicate about a special interest or issue that I care about

\section{Social}

Cronbach's $\alpha=.878$
To stay in touch with my friends and family

To entertain people

To network or to meet new people

To communicate to many people at once, rather than telling one at a time

To feel close to others.

To maintain my social network.

\section{Therapeutic}

Cronbach's $\alpha=.816$
To gain insight into my problems

To ease my distress

To get feedback from others who have similar experiences

To get advice from others

To get more points of view

\section{Directive}

Cronbach's $\alpha=.456$
To share useful information, practical knowledge or skills with others

To influence the way other people think

To motivate other people

To help others 
A few missing data were substituted with group means. Reliability analysis showed excellent internal consistency of the scale as a whole, with Cronbach's $\alpha=.926$. Cronbach's $\alpha$ when each item was excluded ranged from .917 to .929 , indicating excellent internal consistency across items.

Further reliability analysis was conducted for each subscale (see Table 1). The internal consistency reliability ranged from being acceptable to excellent for the first three subscales but was poor for the fourth subscale: Cronbach's $\alpha=.735$ for Self subscale; Cronbach's $\alpha=.878$ for Social subscale; Cronbach's $\alpha=.816$ for Therapeutic subscale; Cronbach's $\alpha=.456$ for Directive subscale.

One explanation for the poor reliability of the Directive subscale is that there are only 4 items for this subscale, each unlikely capturing one unique aspect of directive functions of memory sharing. To include additional items in the subscale, or to make the items focus on one aspect of directive functions (e.g., to impart lessons for current and future behavioral guidance) may increase the internal consistency of the subscale. Alternatively, the four items in the current subscale can be considered separately to understand why people share memories online for various directive functions. It is also important to note that different from directive functions of memory recall that focus on using the past for one's own current and future problem solving (Pillermer, 1998), directive functions of memory sharing focus on using the past to help others. Self-help related functions of memory sharing are captured by the Therapeutic subscale.

Correlations were further computed between the participants' total POMSS score as well as subscale scores and the total number of memories they shared online over a one-week period. The various purposes of online memory sharing were positively associated with the frequency of the sharing activity: total POMSS score, $r=.60, p=.0003$; the Self subscale score, $r=.57, p$ 
$=.0007$; the Social subscale score, $r=.57, p=.0007$; the Therapeutic subscale score, $r=.47, p$ $=.007$; and the Directive subscale score, $r=.67, p<.0001$. Thus, the more participants endorsed the purposes of online memory sharing, the more frequently they shared memories online.

In addition, mean item rating for each subscale was calculated. The Social subscale had the highest mean rating, followed by the Self subscale, the Directive subscale, and the Therapeutic subscale had the lowest mean item rating. Paired comparisons showed that all differences were significant $(p<0.1)$ except the difference between the Directive and Therapeutic subscales $(p=.08)$. It appears that people share memories online most primarily for social functions, followed by self and directive functions, and lastly for emotion regulation and coding. This finding is consistent with prior research showing that relationship maintenance and social connection is the most prominent purpose of memory sharing in everyday life (Alea \& Bluck, 2003; Guan \& Wang, 2020; Kulkofsky et al., 2010).

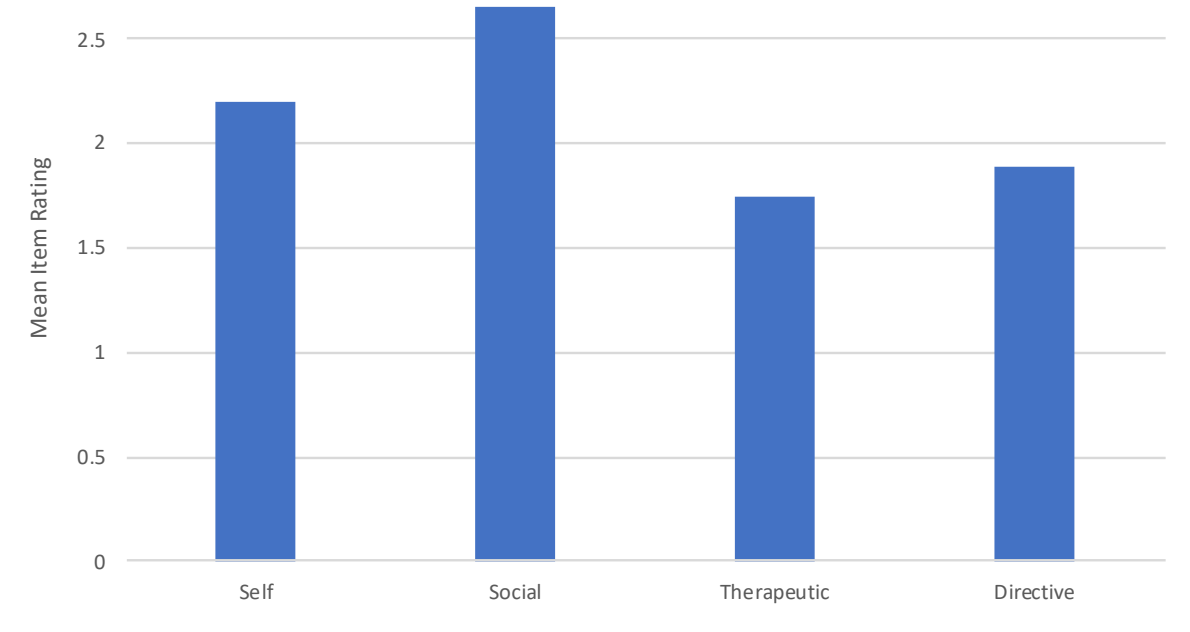

Figure 1. Mean item rating by subscales. 
Taken together, the polit data suggest that the Purposes of Online Memory Sharing Scale (POMSS) is a reliable measure of the reasons for which people share their experiences online. Three of the subscales - Self, Social, and Therapeutic - had acceptable to excellent internal consistency reliability, while the Directive subscale had poor internal consistency and should be used with caution. The positive association between the various purposes of online memory sharing and the frequency of the sharing activity provides support for the validity of the scale. Additional research is needed to test the reliability and validity of the Purposes of Online Memory Sharing Scale (POMSS) and to examine memory sharing in the digital age. 


\section{References}

Alea, N., \& Bluck, S. (2003). Why are you telling me that? A conceptual model of the social function of autobiographical memory. Memory, 11, 165-178.

Baker, J. R., \& Moore, S. M. (2011). Creation and Validation of the Personal Blogging Style Scale. CyberPsychology, Behavior \& Social Networking, 14(6), 379-385.

Bluck, S. (2003). Autobiographical memory: Exploring its functions in everyday life. Memory, 11(2), 113-123.

Guan, L., \& Wang, Q. (2020, April 1). Does sharing memories make us feel closer? The roles of memory type and culture. https://doi.org/10.31234/osf.io/7udy4

Hollenbaugh, E. E. (2011). Motives for maintaining personal journal blogs. CyberPsychology, Behavior \& Social Networking, 14(1/2), 13-20.

Kulkofsky, S., Wang, Q., \& Hou, Y. (2010). Why I remember that: The influence of contextual factors on beliefs about everyday memory. Memory \& Cognition, 38, 461-473.

Lee, D-H, Im, S., \& Taylor, C. R. (2008). Voluntary self-disclosure of information on the internet: A multimethod study of the motivations and consequences of disclosing information on blogs. Psychology \& Marketing, 25, 7, 692-710.

Lenhart, A., \& Fox, S. (2006). Bloggers: A portrait of the Internet's new storytellers. Pew Internet \& American Life. Retrieved October 13, 2011 from http://www.pewinternet.org/ /media//Files/Reports/2006/PIP\%20Bloggers\%20Report\%2 0July\%2019\%202006.pdf.pdf

Lenhart, A., Purcell, K., Smith, A., \& Zichuhr, K. (2010). Social media \& mobile Internet use among teens and young adults. Pew Internet \& American Life. Retrieved December 8, 2011 
from http://pewinternet.org/ /media//Files/Reports/2010/PIP_Social_Media_and_Young_ Adults_Report_Final_with_toplines.pdf.

Nardi, B.A., Schiano, D.J., \& Gumbrecht, M. (2004). Why we blog. Communications of the ACM, 47, 41-6.

Pillemer, D. B. (1998). Momentous events, vivid memories. Cambridge, MA: Harvard University Press.

Schmitt, K. L., Dayanim, S., \& Matthias, S. (2008). Personal homepage construction as an expression of social development. Developmental Psychology, 44(2), 496-506.

Wang, Q. (2013). The autobiographical self in time and culture. Oxford University Press.

Wang, Q., Koh, J. B. K., Song, Q., \& Hou, Y. (2015). Knowledge of memory functions in European and Asian American adults and children: The relation to autobiographical memory. Special issue: Going Global: The Functions of Autobiographical Memory in Cultural Context. Memory, 23, 1, 25-38. doi:10.1080/09658211.2014.930495

Wang, Q., Lee, D., \& Hou, Y. (2017). Externalizing the autobiographical self: Sharing personal memories online facilitated memory retention. Memory, 25, 6, 772-776. doi: 10.1080/09658211.2016.1221115. 
Appendix A

\section{Purposes of Online Memory Sharing Scale}

Why do you post about your experiences online? Indicate how much each of the statements below describes your reasons for sharing your experiences online.

$\begin{array}{ccccc}1 & 2 & 3 & 4 & 5 \\ \text { Not at all } & & & & \text { Exactly my reasons }\end{array}$

1. To express myself

2. To stay in touch with my friends and family

3. To gain insight into my problems

4. To share useful information, practical knowledge or skills with others

5. To entertain people

6. To document my personal experiences

7. To ease my distress

8. To influence the way other people think

9. To network or to meet new people

10. To get attention

11. To communicate to many people at once, rather than telling one at a time

12. To get feedback from others who have similar experiences

13. To motivate other people

14. To record my thoughts and feelings so I can reflect on them

15. To feel close to others.

16. To get advice from others

17. To communicate about a special interest or issue that I care about

18. To help others

19. To maintain my social network.

20. To get more points of view

Color codes:

Blue items $(1,6,10,14,17)$ : Self purpose subscale

Orange items $(2,5,9,11,15,19)$ : Social purpose subscale

Green items $(3,7,12,16,20)$ : Therapeutic purpose subscale

Brown items $(4,8,13,18)$ : Directive purpose subscale 